MATHEMATICS OF COMPUTATION

Volume 75, Number 256, October 2006, Pages 1617-1626

S $0025-5718(06) 01869-2$

Article electronically published on July 11, 2006

\title{
A TWO-GRID DISCRETIZATION METHOD FOR DECOUPLING SYSTEMS OF PARTIAL DIFFERENTIAL EQUATIONS
}

\author{
JICHENG JIN, SHI SHU, AND JINCHAO XU
}

\begin{abstract}
In this paper, we propose a two-grid finite element method for solving coupled partial differential equations, e.g., the Schrödinger-type equation. With this method, the solution of the coupled equations on a fine grid is reduced to the solution of coupled equations on a much coarser grid together with the solution of decoupled equations on the fine grid. It is shown, both theoretically and numerically, that the resulting solution still achieves asymptotically optimal accuracy.
\end{abstract}

\section{INTRODUCTION}

The idea of the two-grid finite element method was originally proposed by $\mathrm{Xu}$ in 19, 20, 21, 22 for discretizing nonsymmetric and indefinite partial differential equations. By employing two finite element spaces of different scales, one coarse space and one fine space, this method was first used for symmetrization of nonsymmetric problems, which reduces the solution of a nonsymmetric problem on a fine grid to the solution of a corresponding (but much smaller) nonsymmetric problem discretized on the coarse grid and the solution of a symmetric positive definite problem on the fine grid. This method was also used for linearization for nonlinear problems [12, 19, 20, for localization and parallelization for solving a large class of partial differential equations [16, 17, 18. There are also many other authors who have used this method for many different applications. See, for example, Axelsson et al. 2, 3, 4, Girault and Lions [7, Layton et al. 9, 10, 11, and Utnes [15.

In this paper, we explore the two-grid idea in a new direction, namely we will use the two-grid discretization method to decouple the systems of partial differential equations. For clarity, we will use a simple model problem of the Schrödinger equation which arises from quantum mechanics to illustrate our idea. Similar to the two-grid discretization method for symmetrization, linearization, localization and parallelization as mentioned above, we use the two-grid method to decouple a system of partial differential equations by first discretizing the original systems of partial differential equations on the coarse grid and then discretizing a decoupled

Received by the editor May 19, 2005 and, in revised form, August 15, 2005.

2000 Mathematics Subject Classification. Primary 65N50, 65N30.

Key words and phrases. Schrödinger type equation, coupled system, finite element method, two-grid.

The research of the first and second authors was supported by NSAF(10376031) and the National Major Key Project for Basic Research and National High-Tech ICF Committee in China.

The research of the third author was supported in part by NSF DMS-0209497 and NSF DMS0215392 and the Furong Scholar Program of Hunan Province through Xiangtan University.

(C)2006 American Mathematical Society 1617

Reverts to public domain 28 years from publication 
system on the fine grid. As a result, the computational complexity of solving, say, a model Schrödinger equation is comparable to solving two decoupled Poisson equations on the same fine grid.

Similar to other applications, the two-grid discretization method for decoupling a system of partial differential equations is not only an efficient numerical method by itself for such applications, but its analysis should provide some insights on how a multiscale idea can be applied for systems of partial differential equations.

The rest of this paper is organized as follows. In section 2, we introduce a model Schrödinger equation used to demonstrate our method. In section 3 , we propose the two-grid finite element algorithms and analyze the convergence. Section 4 is devoted to the presentation of numerical examples showing the effectiveness of our method.

\section{A MOdEL SCHRÖDINGER EQUATION}

The Schrödinger equation is the fundamental equation in quantum mechanics. It also arises in mathematically modelling underwater acoustics, where the Helmholtz equation for the acoustic pressure is transformed into an equation of the same form by applying the so-called "parabolic approximation" [14]. For simplification, here we consider the following boundary value problem of the Schrödinger type:

$$
\begin{array}{ll}
-\Delta \psi(\mathbf{x})+V(\mathbf{x}) \psi(\mathbf{x})=f(\mathbf{x}), & \forall \mathbf{x} \in \Omega, \\
\psi(\mathbf{x})=0, & \forall \mathbf{x} \in \partial \Omega,
\end{array}
$$

where $\Omega \subset \mathbf{R}^{2}$ is a polygonal domain which, for simplicity of exposition, will be assumed to be convex. In general, $f(\mathbf{x})$, the potential function $V(\mathbf{x})$ and unknown function $\psi(\mathbf{x})$ are complex valued.

For any complex-valued function $w(\mathbf{x})$, we denote its real part by $w_{1}(\mathbf{x})$, the imaginary part by $w_{2}(\mathbf{x})$, and the vector function $\left(w_{1}(\mathbf{x}), w_{2}(\mathbf{x})\right)$ by $\mathbf{w}(\mathbf{x})$. Then problem (2.1)-(2.2) is equivalent to the following coupled equations:

$$
\begin{array}{ll}
-\Delta \psi_{1}(\mathbf{x})+V_{1}(\mathbf{x}) \psi_{1}(\mathbf{x})-V_{2}(\mathbf{x}) \psi_{2}(\mathbf{x})=f_{1}(\mathbf{x}), & \forall \mathbf{x} \in \Omega, \\
-\Delta \psi_{2}(\mathbf{x})+V_{1}(\mathbf{x}) \psi_{2}(\mathbf{x})+V_{2}(\mathbf{x}) \psi_{1}(\mathbf{x})=f_{2}(\mathbf{x}), & \forall \mathbf{x} \in \Omega, \\
\psi_{j}(\mathbf{x})=0, \quad j=1,2, & \forall \mathbf{x} \in \partial \Omega .
\end{array}
$$

Let $L^{2}(\Omega)$ be the inner product space with the inner product given by

$$
(u, v)=\int_{\Omega} u(\mathbf{x}) v(\mathbf{x}) d \mathbf{x}
$$

for real-valued and Lebesgue square integrable functions $u(\mathbf{x})$ and $v(\mathbf{x})$, let $H^{m}(\Omega)$ be the standard Sobolev space with a norm given by $\|u\|_{m}^{2}=\sum_{|\alpha| \leq m}\left\|D^{\alpha} u\right\|_{L^{2}(\Omega)}^{2}$ for a real-valued function $u(\mathbf{x})$, and let $H_{0}^{1}(\Omega)$ be the subspace of $H^{1}(\Omega)$ consisting of functions with vanishing trace on $\partial \Omega$. Then the equivalent variational form of (2.3) - (2.5) is defined as follows.

$$
\begin{gathered}
\text { Find } \boldsymbol{\psi} \in H_{0}^{1}(\Omega) \times H_{0}^{1}(\Omega) \text { such that } \\
a(\boldsymbol{\psi}, \mathbf{w})=(\mathbf{f}, \mathbf{w}), \quad \forall \mathbf{w} \in H_{0}^{1}(\Omega) \times H_{0}^{1}(\Omega),
\end{gathered}
$$

where

$$
(\mathbf{f}, \mathbf{w})=\left(f_{1}, w_{1}\right)+\left(f_{2}, w_{2}\right), \quad a(\boldsymbol{\psi}, \mathbf{w})=\widehat{a}(\boldsymbol{\psi}, \mathbf{w})+N(\boldsymbol{\psi}, \mathbf{w})
$$


with

$$
\begin{aligned}
\widehat{a}(\boldsymbol{\psi}, \mathbf{w}) & =\left(\nabla \psi_{1}, \nabla w_{1}\right)+\left(\nabla \psi_{2}, \nabla w_{2}\right) \\
N(\boldsymbol{\psi}, \mathbf{w}) & =\left(V_{1} \psi_{1}, w_{1}\right)-\left(V_{2} \psi_{2}, w_{1}\right)+\left(V_{1} \psi_{2}, w_{2}\right)+\left(V_{2} \psi_{1}, w_{2}\right) .
\end{aligned}
$$

Let the notation " $\lesssim$ " be equivalent to " $\leq C$ " for some positive constant $C$ and $\|\mathbf{w}\|_{m}$ denote $\sqrt{\left\|w_{1}\right\|_{m}^{2}+\left\|w_{2}\right\|_{m}^{2}}$ for any vector function $\mathbf{w}(\mathbf{x})$. Let us first state a simple regularity result.

Theorem 1. Assume that

$$
\mathbf{f} \in L^{2}(\Omega) \times L^{2}(\Omega), \quad \mathbf{V} \in L^{\infty}(\Omega) \times L^{\infty}(\Omega), \quad V_{1}(\mathbf{x}) \geq 0 \text { in } \Omega .
$$

Then the variational problem (2.6) has a unique solution $\boldsymbol{\psi} \in H^{2}(\Omega) \times H^{2}(\Omega)$, and

$$
\|\boldsymbol{\psi}\|_{2} \lesssim\|\mathbf{f}\|_{0}
$$

Proof. From (2.7), we can easily check that

$$
\begin{aligned}
|a(\mathbf{u}, \mathbf{w})| & \lesssim\|\mathbf{u}\|_{1}\|\mathbf{w}\|_{1}, \forall \mathbf{u}, \mathbf{w} \in H_{0}^{1}(\Omega) \times H_{0}^{1}(\Omega), \\
\|\mathbf{w}\|_{1}^{2} & \lesssim a(\mathbf{w}, \mathbf{w}), \quad \forall \mathbf{w} \in H_{0}^{1}(\Omega) \times H_{0}^{1}(\Omega) .
\end{aligned}
$$

Therefore, by the Lax-Milgram theorem, the variation problem (2.6) has a unique solution $\boldsymbol{\psi} \in H_{0}^{1}(\Omega) \times H_{0}^{1}(\Omega)$. Note that $\psi_{1}(\mathbf{x}), \psi_{2}(\mathbf{x})$ are the weak solutions of problem (2.3) - (2.5) . By the regularity theory for elliptic boundary value problems [6], we have

$$
\begin{aligned}
& \left\|\psi_{1}\right\|_{2} \underset{ }{\lesssim}\left\|f_{1}+V_{2} \psi_{2}\right\|_{0} \lesssim\left\|\psi_{2}\right\|_{0}+\left\|f_{1}\right\|_{0}, \\
& \left\|\psi_{2}\right\|_{2} \lesssim\left\|f_{2}-V_{2} \psi_{1}\right\|_{0} \lesssim\left\|\psi_{1}\right\|_{0}+\left\|f_{2}\right\|_{0} .
\end{aligned}
$$

The above two inequalities imply that

$$
\|\boldsymbol{\psi}\|_{2} \lesssim\|\boldsymbol{\psi}\|_{0}+\|\mathbf{f}\|_{0} .
$$

From (2.6) we have

$$
\|\boldsymbol{\psi}\|_{1}^{2} \lesssim a(\boldsymbol{\psi}, \boldsymbol{\psi})=(\mathbf{f}, \boldsymbol{\psi}) \lesssim\|\boldsymbol{\psi}\|_{0}\|\mathbf{f}\|_{0}
$$

and then

$$
\|\boldsymbol{\psi}\|_{1} \lesssim\|\mathbf{f}\|_{0} .
$$

Therefore, (2.8) follows from (2.9) and the above inequality.

Let $T_{h}$ be a quasi-uniform triangulation of $\Omega$ with mesh size $h>0$, and let $S_{0}^{h} \subset H_{0}^{1}(\Omega)$ be the corresponding piecewise linear polynomial space. Then the finite element approximation of problem (2.6) is defined as follows.

Find $\boldsymbol{\psi}_{h} \in S_{0}^{h} \times S_{0}^{h}$ such that

$$
a\left(\boldsymbol{\psi}_{h}, \mathbf{w}_{h}\right)=\left(\mathbf{f}, \mathbf{w}_{h}\right), \quad \forall \mathbf{w}_{h} \in S_{0}^{h} \times S_{0}^{h} .
$$

As shown in the following theory, the error analysis of the above finite element discretization can be obtained by standard techniques.

Theorem 2. Under the assumption (2.7), $\boldsymbol{\psi}_{h}$ has the error estimate

$$
\left\|\boldsymbol{\psi}-\boldsymbol{\psi}_{h}\right\|_{s} \lesssim h^{2-s}\|\boldsymbol{\psi}\|_{2}, \quad s=0,1 .
$$


Proof. Let $\mathbf{e}_{h}=\boldsymbol{\psi}-\boldsymbol{\psi}_{h}$. Then it follows from (2.6) and (2.10) that

$$
a\left(\mathbf{e}_{h}, \mathbf{w}_{h}\right)=0, \quad \forall \mathbf{w}_{h} \in S_{0}^{h} \times S_{0}^{h} .
$$

Let $\boldsymbol{\psi}^{I} \in S_{0}^{h} \times S_{0}^{h}$ be the interpolation of $\boldsymbol{\psi}$. Then

$$
\left\|\mathbf{e}_{h}\right\|_{1}^{2} \lesssim a\left(\mathbf{e}_{h}, \mathbf{e}_{h}\right)=a\left(\mathbf{e}_{h}, \boldsymbol{\psi}-\boldsymbol{\psi}^{I}\right) \lesssim\left\|\mathbf{e}_{h}\right\|_{1}\left\|\boldsymbol{\psi}-\boldsymbol{\psi}^{I}\right\|_{1},
$$

which implies that

$$
\left\|\mathbf{e}_{h}\right\|_{1} \lesssim\left\|\boldsymbol{\psi}-\boldsymbol{\psi}^{I}\right\|_{1} \lesssim h\|\boldsymbol{\psi}\|_{2} .
$$

We consider the auxiliary problem of (2.3)-(2.5):

$$
\begin{array}{ll}
-\Delta u_{1}(\mathbf{x})+V_{1}(\mathbf{x}) u_{1}(\mathbf{x})+V_{2}(\mathbf{x}) u_{2}(\mathbf{x})=g_{1}(\mathbf{x}), & \forall \mathbf{x} \in \Omega, \\
-\Delta u_{2}(\mathbf{x})+V_{1}(\mathbf{x}) u_{2}(\mathbf{x})-V_{2}(\mathbf{x}) u_{1}(\mathbf{x})=g_{2}(\mathbf{x}), & \forall \mathbf{x} \in \Omega, \\
u_{j}(\mathbf{x})=0, \quad j=1,2, & \forall \mathbf{x} \in \partial \Omega .
\end{array}
$$

Then similar to Theorem 1, for any $\mathbf{g} \in L^{2}(\Omega) \times L^{2}(\Omega)$ there exists a unique solution $\mathbf{u} \in\left(H^{2}(\Omega) \cap H_{0}^{1}(\Omega)\right) \times\left(H^{2}(\Omega) \bigcap H_{0}^{1}(\Omega)\right)$ such that

$$
a(\mathbf{w}, \mathbf{u})=(\mathbf{g}, \mathbf{w}), \quad \forall \mathbf{w} \in H_{0}^{1}(\Omega) \times H_{0}^{1}(\Omega),
$$

and

$$
\|\mathbf{u}\|_{2} \lesssim\|\mathbf{g}\|_{0} .
$$

Take $\mathbf{g}=\mathbf{e}_{h}$ in (2.17) and let $\mathbf{u}^{I} \in S_{0}^{h} \times S_{0}^{h}$ be the interpolation of $\mathbf{u}$. Then we have

$$
\begin{aligned}
\left\|\mathbf{e}_{h}\right\|_{0}^{2} & =a\left(\mathbf{e}_{h}, \mathbf{u}\right)=a\left(\mathbf{e}_{h}, \mathbf{u}-\mathbf{u}^{I}\right) \\
& \lesssim\left\|\mathbf{e}_{h}\right\|_{1}\left\|\mathbf{u}-\mathbf{u}^{I}\right\|_{1} \lesssim h\left\|\mathbf{e}_{h}\right\|_{1}\|\mathbf{u}\|_{2} \lesssim h\left\|\mathbf{e}_{h}\right\|_{1}\left\|\mathbf{e}_{h}\right\|_{0},
\end{aligned}
$$

which implies that

$$
\left\|\mathbf{e}_{h}\right\|_{0} \lesssim h\left\|\mathbf{e}_{h}\right\|_{1} .
$$

Therefore, (2.11) follows from (2.12) and (2.19).

\section{A NEW TWO-GRID FINITE ELEMENT METHOD}

The finite element discretization (2.10) apparently corresponds to a coupled system of equations in the general case that the potential function $V(\mathbf{x})$ given in (2.1) is complex valued. In order to reduce the computational cost, following $\mathrm{Xu}$ 12, 19, 20, we introduce another finite element space $S_{0}^{H}\left(\subset S_{0}^{h} \subset H_{0}^{1}(\Omega)\right)$ defined on a coarser quasi-uniform triangulation (with meshsize $H>h$ ) of $\Omega$, and propose the following algorithm.

\section{Algorithm A1.}

Step 1. Find $\boldsymbol{\psi}_{H} \in S_{0}^{H} \times S_{0}^{H}$ such that

Step 2. Find $\boldsymbol{\psi}_{h}^{*} \in S_{0}^{h} \times S_{0}^{h}$ such that

$$
\widehat{a}\left(\boldsymbol{\psi}_{h}^{*}, \mathbf{w}_{h}\right)=\left(\mathbf{f}, \mathbf{w}_{h}\right)-N\left(\boldsymbol{\psi}_{H}, \mathbf{w}_{h}\right), \quad \forall \mathbf{w}_{h} \in S_{0}^{h} \times S_{0}^{h} .
$$


We note that the linear system in Step 2 is a decoupled system which involves only two separate Poisson equations, and only on the coarser space a coupled system needs to be solved in Step 1. As the following theorem shows, $\boldsymbol{\psi}_{h}^{*}$ can reach the optimal accuracy in $H^{1}$-norm if the coarse meshsize $H$ is taken to be $\sqrt{h}$. Because the dimension of $S_{0}^{H}$ is much smaller than the dimension of $S_{0}^{h}$, the efficiency of the algorithm is then evident.

Theorem 3. Under the assumption (2.7), $\boldsymbol{\psi}_{h}^{*}$ hass the following error estimate:

$$
\left\|\boldsymbol{\psi}_{h}-\boldsymbol{\psi}_{h}^{*}\right\|_{1} \lesssim H^{2}
$$

Consequently,

$$
\left\|\boldsymbol{\psi}-\boldsymbol{\psi}_{h}^{*}\right\|_{1} \lesssim h+H^{2}
$$

namely, $\boldsymbol{\psi}_{h}^{*}$ has the same accuracy as $\boldsymbol{\psi}_{h}$ in $H^{1}$-norm if $H=\sqrt{h}$.

Proof. Let $\mathbf{e}_{h}=\boldsymbol{\psi}-\boldsymbol{\psi}_{h}, \widehat{\mathbf{e}}_{h}=\boldsymbol{\psi}_{h}-\boldsymbol{\psi}_{h}^{*}$. Then from (2.10) and (3.2) we get

$$
\widehat{a}\left(\widehat{\mathbf{e}}_{h}, \mathbf{w}_{h}\right)+N\left(\boldsymbol{\psi}_{h}-\boldsymbol{\psi}_{H}, \mathbf{w}_{h}\right)=0, \forall \mathbf{w}_{h} \in S_{0}^{h} \times S_{0}^{h} .
$$

By taking $\mathbf{w}_{h}=\widehat{\mathbf{e}}_{h}$ in the above equality, we have

$$
\left\|\widehat{\mathbf{e}}_{h}\right\|_{1}^{2} \lesssim \widehat{a}\left(\widehat{\mathbf{e}}_{h}, \widehat{\mathbf{e}}_{h}\right) \lesssim\left\|\boldsymbol{\psi}_{h}-\boldsymbol{\psi}_{H}\right\|_{0}\left\|\widehat{\mathbf{e}}_{h}\right\|_{0},
$$

and then

$$
\left\|\widehat{\mathbf{e}}_{h}\right\|_{1} \lesssim\left\|\psi_{h}-\psi_{H}\right\|_{0}
$$

From Theorem 2 we get

$$
\left\|\boldsymbol{\psi}_{h}-\boldsymbol{\psi}_{H}\right\|_{0} \leq\left\|\boldsymbol{\psi}-\boldsymbol{\psi}_{h}\right\|_{0}+\left\|\boldsymbol{\psi}-\boldsymbol{\psi}_{H}\right\|_{0} \lesssim h^{2}+H^{2}
$$

Therefore, (3.3) follows from (3.6) and the above inequality. Also, (3.4) follows from (2.11), (3.3) and the following inequality:

$$
\left\|\boldsymbol{\psi}-\boldsymbol{\psi}_{h}^{*}\right\|_{1} \leq\left\|\boldsymbol{\psi}-\boldsymbol{\psi}_{h}\right\|_{1}+\left\|\widehat{\mathbf{e}}_{h}\right\|_{1} .
$$

Algorithm A1 can be improved in a successive fashion as follows.

Algorithm A2. Let $\boldsymbol{\psi}_{h}^{0}=\mathbf{0}$. Assume that $\boldsymbol{\psi}_{h}^{k} \in S_{0}^{h} \times S_{0}^{h}$ has been obtained, then $\boldsymbol{\psi}_{h}^{k+1} \in S_{0}^{h} \times S_{0}^{h}$ is defined as follows:

Step 1. Find $\mathbf{e}_{H} \in S_{0}^{H} \times S_{0}^{H}$ such that

$$
a\left(\mathbf{e}_{H}, \boldsymbol{\chi}\right)=(\mathbf{f}, \boldsymbol{\chi})-a\left(\boldsymbol{\psi}_{h}^{k}, \boldsymbol{\chi}\right), \quad \forall \boldsymbol{\chi} \in S_{0}^{H} \times S_{0}^{H} .
$$

Step 2. Find $\boldsymbol{\psi}_{h}^{k+1} \in S_{0}^{h} \times S_{0}^{h}$ such that

$$
\widehat{a}\left(\boldsymbol{\psi}_{h}^{k+1}, \mathbf{w}_{h}\right)=\left(\mathbf{f}, \mathbf{w}_{h}\right)-N\left(\boldsymbol{\psi}_{h}^{k}+\mathbf{e}_{H}, \mathbf{w}_{h}\right), \quad \forall \mathbf{w}_{h} \in S_{0}^{h} \times S_{0}^{h} .
$$

Theorem 4. Under the assumption (2.7), $\boldsymbol{\psi}_{h}^{k}$ admits the following error estimate:

$$
\left\|\boldsymbol{\psi}_{h}-\boldsymbol{\psi}_{h}^{k}\right\|_{1} \lesssim H^{k+1}, \quad k \geq 1 .
$$

Consequently,

$$
\left\|\boldsymbol{\psi}-\boldsymbol{\psi}_{h}^{k}\right\|_{1} \lesssim h+H^{k+1}, \quad k \geq 1
$$

namely, $\boldsymbol{\psi}_{h}^{k}, k \geq 1$, has the same accuracy as $\boldsymbol{\psi}_{h}$ in $H^{1}$-norm if $H=h^{\frac{1}{k+1}}$. 
Proof. From (2.10) and (3.8), we have

$$
\widehat{a}\left(\boldsymbol{\psi}_{h}-\boldsymbol{\psi}_{h}^{k+1}, \mathbf{w}_{h}\right)=-N\left(\boldsymbol{\psi}_{h}-\left(\boldsymbol{\psi}_{h}^{k}+\mathbf{e}_{H}\right), \mathbf{w}_{h}\right), \forall \mathbf{w}_{h} \in S_{0}^{h} \times S_{0}^{h},
$$

which, by taking $\mathbf{w}_{h}=\boldsymbol{\psi}_{h}-\boldsymbol{\psi}_{h}^{k+1}$, gives

$$
\left\|\boldsymbol{\psi}_{h}-\boldsymbol{\psi}_{h}^{k+1}\right\|_{1}^{2} \lesssim \widehat{a}\left(\boldsymbol{\psi}_{h}-\boldsymbol{\psi}_{h}^{k+1}, \boldsymbol{\psi}_{h}-\boldsymbol{\psi}_{h}^{k+1}\right) \lesssim\left\|\boldsymbol{\psi}_{h}-\left(\boldsymbol{\psi}_{h}^{k}+\mathbf{e}_{H}\right)\right\|_{0}\left\|\boldsymbol{\psi}_{h}-\boldsymbol{\psi}_{h}^{k+1}\right\|_{0},
$$

and then

$$
\left\|\boldsymbol{\psi}_{h}-\boldsymbol{\psi}_{h}^{k+1}\right\|_{1} \lesssim\left\|\boldsymbol{\psi}_{h}-\left(\boldsymbol{\psi}_{h}^{k}+\mathbf{e}_{H}\right)\right\|_{0} .
$$

It follows from (2.10) and (3.7) that

$$
a\left(\boldsymbol{\psi}_{h}-\left(\boldsymbol{\psi}_{h}^{k}+\mathbf{e}_{H}\right), \boldsymbol{\chi}\right)=0, \forall \boldsymbol{\chi} \in S_{0}^{H} \times S_{0}^{H} .
$$

Thus

$$
\begin{aligned}
\left\|\boldsymbol{\psi}_{h}-\left(\boldsymbol{\psi}_{h}^{k}+\mathbf{e}_{H}\right)\right\|_{1}^{2} & \lesssim a\left(\boldsymbol{\psi}_{h}-\left(\boldsymbol{\psi}_{h}^{k}+\mathbf{e}_{H}\right), \boldsymbol{\psi}_{h}-\left(\boldsymbol{\psi}_{h}^{k}+\mathbf{e}_{H}\right)\right) \\
& =a\left(\boldsymbol{\psi}_{h}-\left(\boldsymbol{\psi}_{h}^{k}+\mathbf{e}_{H}\right), \boldsymbol{\psi}_{h}-\boldsymbol{\psi}_{h}^{k}\right) \\
& \lesssim\left\|\boldsymbol{\psi}_{h}-\left(\boldsymbol{\psi}_{h}^{k}+\mathbf{e}_{H}\right)\right\|_{1}\left\|\boldsymbol{\psi}_{h}-\boldsymbol{\psi}_{h}^{k}\right\|_{1},
\end{aligned}
$$

and then

$$
\left\|\boldsymbol{\psi}_{h}-\left(\boldsymbol{\psi}_{h}^{k}+\mathbf{e}_{H}\right)\right\|_{1} \lesssim\left\|\boldsymbol{\psi}_{h}-\boldsymbol{\psi}_{h}^{k}\right\|_{1} .
$$

Let $\mathbf{u}$ be the solution of problem (2.17) with $\mathbf{g}=\boldsymbol{\psi}_{h}-\left(\boldsymbol{\psi}_{h}^{k}+\mathbf{e}_{H}\right)$ and let $\mathbf{u}^{I} \in S_{0}^{H} \times S_{0}^{H}$ be the interpolation of $\mathbf{u}$. Then according to (2.18) and (3.13), we have

$$
\begin{aligned}
\left\|\boldsymbol{\psi}_{h}-\left(\boldsymbol{\psi}_{h}^{k}+\mathbf{e}_{H}\right)\right\|_{0}^{2} & =a\left(\boldsymbol{\psi}_{h}-\left(\boldsymbol{\psi}_{h}^{k}+\mathbf{e}_{H}\right), \mathbf{u}\right)=a\left(\boldsymbol{\psi}_{h}-\left(\boldsymbol{\psi}_{h}^{k}+\mathbf{e}_{H}\right), \mathbf{u}-\mathbf{u}^{I}\right) \\
& \lesssim\left\|\boldsymbol{\psi}_{h}-\left(\boldsymbol{\psi}_{h}^{k}+\mathbf{e}_{H}\right)\right\|_{1}\left\|\mathbf{u}-\mathbf{u}^{I}\right\|_{1} \\
& \lesssim H\left\|\boldsymbol{\psi}_{h}-\left(\boldsymbol{\psi}_{h}^{k}+\mathbf{e}_{H}\right)\right\|_{1}\|\mathbf{u}\|_{2} \\
& \lesssim H\left\|\boldsymbol{\psi}_{h}-\left(\boldsymbol{\psi}_{h}^{k}+\mathbf{e}_{H}\right)\right\|_{1}\left\|\boldsymbol{\psi}_{h}-\left(\boldsymbol{\psi}_{h}^{k}+\mathbf{e}_{H}\right)\right\|_{0},
\end{aligned}
$$

which implies that

$$
\left\|\boldsymbol{\psi}_{h}-\left(\boldsymbol{\psi}_{h}^{k}+\mathbf{e}_{H}\right)\right\|_{0} \lesssim H\left\|\boldsymbol{\psi}_{h}-\left(\boldsymbol{\psi}_{h}^{k}+\mathbf{e}_{H}\right)\right\|_{1} .
$$

Therefore, from (3.12), (3.15), and (3.14), we have

$$
\left\|\boldsymbol{\psi}_{h}-\boldsymbol{\psi}_{h}^{k}\right\|_{1} \lesssim H\left\|\boldsymbol{\psi}_{h}-\boldsymbol{\psi}_{h}^{k-1}\right\|_{1} \lesssim H^{k-1}\left\|\boldsymbol{\psi}_{h}-\boldsymbol{\psi}_{h}^{1}\right\|_{1}, \quad k \geq 1 .
$$

Note that $\boldsymbol{\psi}_{h}^{1}$ is the solution $\boldsymbol{\psi}_{h}^{*}$ obtained by Algorithm A1, thus, (3.9) follows from (3.16) and (3.3). Additionally, (3.10) follows from (3.9), (2.11), and the following inequality:

$$
\left\|\boldsymbol{\psi}-\boldsymbol{\psi}_{h}^{k}\right\|_{1} \leq\left\|\boldsymbol{\psi}-\boldsymbol{\psi}_{h}\right\|_{1}+\left\|\boldsymbol{\psi}_{h}-\boldsymbol{\psi}_{h}^{k}\right\|_{1}
$$


According to Theorem 4, it suffices to take $H=h^{\frac{1}{k+1}}$ to obtain the optimal approximation in $H^{1}$-norm. Therefore, the dimension of $S_{0}^{H}$ can be much smaller than the dimension of $S_{0}^{h}$, and thus the dominated part of the work in Algorithm A2 is to solve two separate Laplacian systems in Step 2, which is much easier solved than the coupled system in (2.10).

\section{NumERICAL EXAMPLES}

In this section we will demonstrate the efficiency of our algorithms proposed in section 3 by two numerical examples. Here, we consider the following boundary value problem of the Schrödinger type:

$$
\begin{array}{ll}
-\Delta \psi(\mathbf{x})+V(\mathbf{x}) \psi(\mathbf{x})=f(\mathbf{x}), & \forall \mathbf{x} \in \Omega, \\
\psi(\mathbf{x})=0, & \forall \mathbf{x} \in \partial \Omega,
\end{array}
$$

where $\Omega=(0,1)^{2}, V(\mathbf{x})=1+i$.

Example 1. $f(\mathbf{x})$ is so chosen that $\psi(\mathbf{x})=(0.5+i) \sin (\pi x) \sin (\pi y)$ is the exact solution.

The domain $\Omega$ is uniformly divided by two nested triangulations of mesh size $H$ and $h$, respectively, $S_{0}^{H}$ and $S_{0}^{h}$ are the corresponding piecewise linear finite element spaces. The standard finite element solution $\boldsymbol{\psi}_{h}$ on different meshes is first computed by (2.10), and the numerical results are listed in Table 1, which shows that $\left\|\boldsymbol{\psi}-\boldsymbol{\psi}_{h}\right\|_{s} \approx O\left(h^{2-s}\right), s=0,1$. For $H=1 / 4,1 / 8$, and $h=H^{2}$, $\boldsymbol{\psi}_{h}^{*}$ is computed by Algorithm A1, and the numerical results are listed in Table 2. We can see that $\left\|\boldsymbol{\psi}-\boldsymbol{\psi}_{h}^{*}\right\|_{1} \approx O\left(H^{2}\right)$, which coincides with the theoretical result obtained in Theorem [3. For $H=1 / 4$ and $h=H^{3}, \boldsymbol{\psi}_{h}^{2}$ is computed by Algorithm A2, and its error together with the errors of $\boldsymbol{\psi}_{h}^{1}, \boldsymbol{\psi}_{h}^{3}$ and the standard finite element solution $\psi_{h}$ are listed in Table 3. Just as Theorem 4 shows, in case that $h=H^{3}, \boldsymbol{\psi}_{h}^{2}$ has the same accuracy as $\boldsymbol{\psi}_{h}$ in $H^{1}$-norm. Finally, for fixed

TABLE 1. Errors of $\boldsymbol{\psi}_{h}$ in $H^{1}$ and $L^{2}$ norms.

\begin{tabular}{|c|cc|}
\hline Mesh & $\left\|\boldsymbol{\psi}-\boldsymbol{\psi}_{h}\right\|_{1}$ & $\left\|\boldsymbol{\psi}-\boldsymbol{\psi}_{h}\right\|_{0}$ \\
\hline$h=1 / 16$ & $2.43 \mathrm{D}-01$ & $5.78 \mathrm{D}-03$ \\
$h=1 / 32$ & $1.22 \mathrm{D}-01$ & $1.45 \mathrm{D}-03$ \\
$h=1 / 64$ & $6.09 \mathrm{D}-02$ & $3.63 \mathrm{D}-04$ \\
\hline
\end{tabular}

TABLE 2. Errors of $\boldsymbol{\psi}_{h}^{*}$ with $h=H^{2}$.

\begin{tabular}{|c|cc|}
\hline Mesh & $\left\|\boldsymbol{\psi}-\boldsymbol{\psi}_{h}^{*}\right\|_{1}$ & $\left\|\boldsymbol{\psi}-\boldsymbol{\psi}_{h}^{*}\right\|_{0}$ \\
\hline$H=1 / 4, h=1 / 16$ & $2.44 \mathrm{D}-01$ & $4.70 \mathrm{D}-03$ \\
$H=1 / 8, h=1 / 64$ & $6.13 \mathrm{D}-02$ & $1.22 \mathrm{D}-03$ \\
\hline
\end{tabular}

TABLE 3. Errors of $\boldsymbol{\psi}_{h}^{2}, \boldsymbol{\psi}_{h}^{1}$ and $\boldsymbol{\psi}_{h} ; H=1 / 4, h=H^{3}$.

\begin{tabular}{|c|cc|}
\hline & $H^{1}$-norm & $L^{2}$-norm \\
\hline $\boldsymbol{\psi}-\boldsymbol{\psi}_{H}$ & $9.42 \mathrm{D}-01$ & $8.56 \mathrm{D}-02$ \\
$\boldsymbol{\psi}-\boldsymbol{\psi}_{h}^{1}$ & $6.60 \mathrm{D}-02$ & $5.24 \mathrm{D}-03$ \\
$\boldsymbol{\psi}-\boldsymbol{\psi}_{h}^{2}$ & $6.09 \mathrm{D}-02$ & $3.70 \mathrm{D}-04$ \\
$\boldsymbol{\psi}-\boldsymbol{\psi}_{h}^{3}$ & $6.09 \mathrm{D}-02$ & $3.63 \mathrm{D}-04$ \\
$\boldsymbol{\psi}-\boldsymbol{\psi}_{h}$ & $6.09 \mathrm{D}-02$ & $3.63 \mathrm{D}-04$ \\
\hline
\end{tabular}


TABLE 4. Errors between $\boldsymbol{\psi}_{h}$ and $\boldsymbol{\psi}_{h}^{k}, k=1,2,3 . h=1 / 64$ is fixed.

\begin{tabular}{|c|cccccc|}
\hline$H$ & $\left\|\boldsymbol{\psi}_{h}-\boldsymbol{\psi}_{h}^{1}\right\|_{1}$ & Ratio & $\left\|\boldsymbol{\psi}_{h}-\boldsymbol{\psi}_{h}^{2}\right\|_{1}$ & Ratio & $\left\|\boldsymbol{\psi}_{h}-\boldsymbol{\psi}_{h}^{3}\right\|_{1}$ & Ratio \\
\hline $1 / 4$ & $2.52 \mathrm{D}-02$ & & $3.10 \mathrm{D}-04$ & & $4.00 \mathrm{D}-06$ & \\
$1 / 8$ & $6.55 \mathrm{D}-03$ & 3.8 & $2.15 \mathrm{D}-05$ & 14.4 & $7.58 \mathrm{D}-08$ & 52.8 \\
$1 / 16$ & $1.58 \mathrm{D}-03$ & 4.1 & $1.27 \mathrm{D}-06$ & 16.9 & $1.11 \mathrm{D}-09$ & 68.3 \\
$1 / 32$ & $3.17 \mathrm{D}-04$ & 5.0 & $5.38 \mathrm{D}-08$ & 23.6 & $1.19 \mathrm{D}-11$ & 93.3 \\
\hline
\end{tabular}

TABLE 5. Errors between $\boldsymbol{\psi}_{h}$ and $\boldsymbol{\psi}_{h}^{k}, k=1,2,3 . h=1 / 64$ is fixed.

\begin{tabular}{|c|cccccc|}
\hline$H$ & $\left\|\boldsymbol{\psi}_{h}-\boldsymbol{\psi}_{h}^{1}\right\|_{0}$ & Ratio & $\left\|\boldsymbol{\psi}_{h}-\boldsymbol{\psi}_{h}^{2}\right\|_{0}$ & Ratio & $\left\|\boldsymbol{\psi}_{h}-\boldsymbol{\psi}_{h}^{3}\right\|_{0}$ & Ratio \\
\hline $1 / 4$ & $5.46 \mathrm{D}-03$ & & $6.65 \mathrm{D}-05$ & & $8.50 \mathrm{D}-07$ & \\
$1 / 8$ & $1.42 \mathrm{D}-03$ & 3.8 & $4.60 \mathrm{D}-06$ & 14.5 & $1.60 \mathrm{D}-08$ & 53.1 \\
$1 / 16$ & $3.43 \mathrm{D}-04$ & 4.1 & $2.72 \mathrm{D}-07$ & 16.9 & $2.33 \mathrm{D}-10$ & 68.7 \\
$1 / 32$ & $6.89 \mathrm{D}-05$ & 5.0 & $1.15 \mathrm{D}-08$ & 23.7 & $2.54 \mathrm{D}-12$ & 91.7 \\
\hline
\end{tabular}

$h=1 / 64$ and different $H=1 / 4,1 / 8,1 / 16,1 / 32, \psi_{h}^{k}, k=1,2,3$, are computed by Algorithm A2. The errors between the standard finite element solution $\boldsymbol{\psi}_{h}$ and $\boldsymbol{\psi}_{h}^{k}$ are listed in Table 4 for $H^{1}$-norm and Table 5 for $L^{2}$-norm, and the ratios of the errors are also listed. From Table 4, we can see that $\left\|\boldsymbol{\psi}_{h}-\boldsymbol{\psi}_{h}^{1}\right\|_{1} \approx O\left(H^{2}\right)$, which verifies the theoretical results (3.9) with $k=1$ in Theorem 4. Note that $\boldsymbol{\psi}_{h}^{1}$ is just $\boldsymbol{\psi}_{h}^{*}$ computed by Algorithm A1, so the theoretical results (3.3) in Theorem 3 are also valid. However, for $k \geq 2,\left\|\boldsymbol{\psi}_{h}-\boldsymbol{\psi}_{h}^{k}\right\|_{1}$ is decreasing rather faster than $O\left(H^{k+1}\right)$; it seems as if $\left\|\boldsymbol{\psi}_{h}-\boldsymbol{\psi}_{h}^{k}\right\|_{1} /\left\|\boldsymbol{\psi}_{h}-\boldsymbol{\psi}_{h}^{k-1}\right\|_{1} \approx O\left(H^{2}\right)$, therefore, $\left\|\boldsymbol{\psi}_{h}-\boldsymbol{\psi}_{h}^{k}\right\|_{1} \approx O\left(H^{2 k}\right)$. This suggests that the error bound obtained in (3.9) may not be optimal, but this error bound is the best one we are able to obtain so far. We plan to make further theoretical investigation in a future work to see if further improvements can be made on this type of error estimate. As to the errors in $L^{2}$-norm, Table 5 shows that $\left\|\boldsymbol{\psi}_{h}-\boldsymbol{\psi}_{h}^{k}\right\|_{0}$ has the same order of convergence as $\left\|\boldsymbol{\psi}_{h}-\boldsymbol{\psi}_{h}^{k}\right\|_{1}$. Therefore, both Algorithms A1 and A2 are not optimal in $L^{2}$-norm, which is a typical behavior of this kind of two-grid method.

In order to make more observations about the behavior of $\left\|\boldsymbol{\psi}_{h}-\boldsymbol{\psi}_{h}^{k}\right\|_{s}, s=0,1$, we consider another example as follows.

TABLE 6 . Errors between $\boldsymbol{\psi}_{h}$ and $\boldsymbol{\psi}_{h}^{k}, k=1,2,3 . h=1 / 64$ is fixed.

\begin{tabular}{|c|cccccc|}
\hline$H$ & $\left\|\boldsymbol{\psi}_{h}-\boldsymbol{\psi}_{h}^{1}\right\|_{1}$ & Ratio & $\left\|\boldsymbol{\psi}_{h}-\boldsymbol{\psi}_{h}^{2}\right\|_{1}$ & Ratio & $\left\|\boldsymbol{\psi}_{h}-\boldsymbol{\psi}_{h}^{3}\right\|_{1}$ & Ratio \\
\hline $1 / 4$ & $1.17 \mathrm{D}-01$ & & $1.44 \mathrm{D}-03$ & & $1.84 \mathrm{D}-05$ & \\
$1 / 8$ & $3.03 \mathrm{D}-02$ & 3.9 & $9.83 \mathrm{D}-05$ & 14.7 & $3.42 \mathrm{D}-07$ & 53.8 \\
$1 / 16$ & $7.30 \mathrm{D}-03$ & 4.2 & $5.79 \mathrm{D}-06$ & 17.0 & $4.96 \mathrm{D}-09$ & 69.0 \\
$1 / 32$ & $1.46 \mathrm{D}-03$ & 5.0 & $2.45 \mathrm{D}-07$ & 23.6 & $4.98 \mathrm{D}-11$ & 99.6 \\
\hline
\end{tabular}

TABLE 7. Errors between $\boldsymbol{\psi}_{h}$ and $\boldsymbol{\psi}_{h}^{k}, k=1,2,3 . h=1 / 64$ is fixed.

\begin{tabular}{|c|cccccc|}
\hline$H$ & $\left\|\boldsymbol{\psi}_{h}-\boldsymbol{\psi}_{h}^{\top}\right\|_{0}$ & Ratio & $\left\|\boldsymbol{\psi}_{h}-\boldsymbol{\psi}_{h}^{2}\right\|_{0}$ & Ratio & $\left\|\boldsymbol{\psi}_{h}-\boldsymbol{\psi}_{h}^{3}\right\|_{0}$ & Ratio \\
\hline $1 / 4$ & $2.54 \mathrm{D}-02$ & & $3.09 \mathrm{D}-04$ & & $3.92 \mathrm{D}-06$ & \\
$1 / 8$ & $6.58 \mathrm{D}-03$ & 3.9 & $2.11 \mathrm{D}-05$ & 14.6 & $7.24 \mathrm{D}-08$ & 54.1 \\
$1 / 16$ & $1.59 \mathrm{D}-03$ & 4.1 & $1.24 \mathrm{D}-06$ & 17.1 & $1.05 \mathrm{D}-09$ & 69.0 \\
$1 / 32$ & $3.18 \mathrm{D}-04$ & 5.0 & $5.25 \mathrm{D}-08$ & 23.6 & $1.09 \mathrm{D}-11$ & 96.3 \\
\hline
\end{tabular}


Example 2. $f(\mathbf{x})$ is so chosen that $\psi(\mathbf{x})=(1+20 i) x(1-x) \sin (\pi y)$ is the exact solution.

Similar to Example 1, for fixed $h=1 / 64$ and different $H=1 / 4,1 / 8,1 / 16,1 / 32$, $\boldsymbol{\psi}_{h}^{k}, k=1,2,3$, are computed by Algorithm A2, the errors between the standard finite element solution $\boldsymbol{\psi}_{h}$ and $\boldsymbol{\psi}_{h}^{k}$ are listed in Tables[6] and 7 . From these numerical results, we can also have the same conclusions about $\boldsymbol{\psi}_{h}^{k}$ as in Example 1.

\section{Concluding Remarks}

Using the Schrödinger equation as an illustration, we presented in this paper a new two-grid discretization technique to decouple systems of partial differential equations. This is a new application of the two-grid idea. This two-grid decoupling technique can obviously be extended in many different ways, for example for different discretizations such as finite volume and finite difference methods, for other types of systems of partial differential equations. We hope this short paper will trigger some subsequent works on this new idea.

\section{ACKNOWLEDGMENTS}

The authors wish to thank Mrs. Min Tan for her help on numerical experiments.

\section{REFERENCES}

[1] T. Arbogast, Analysis of a two-scale, locally conservative subgrid upscaling for elliptic problems, SIAM J. Numer. Anal., 42, 2(2004), 576-598. MR2084227 (2005h:65205)

[2] O. Axelsson and I.E. Kaporin, Minimum residul adaptive multilevel finite element procedure for the solution of nonlinear stationary problems, SIAM J. Numer. Anal., 35, 3(1998), 1213-1229. MR1619895 (99d:65171)

[3] O. Axelsson and A. Padiy, On a two level Newton type procedure applied for solving nonlinear elasticity problems, Int. J. Numer. Meth. Engng., 49 (2000), 1479-1493. MR.1797720 (2001i:74084)

[4] O. Axelsson and W. Layton, A two-level discretization of nonlinear boundary value problems, SIAM J. Numer. Anal., 33 (1996), 2359-2374. MR1427468 (98c:65181)

[5] S.N. Chandler-Wilde, M. Rahman and C. R. Ross, A fast two-grid and finite section method for a class of integral equations on the real line with application to an acoustic scattering problem in the half-plane, Numer. Math. 93 (2002), 1-51. MR1938321 (2003h:65177)

[6] D. Gilbarg and N. S. Trudinger, Elliptic partial differential equations of second order, Springer-Verlag, 1977. MR 0473443 (57:13109)

[7] V. Girault and J. L. Lions, Two-grid finite-element schemes for the transient Navier-Stokes problem. Mathematical modelling and numerical analysis, M2AN, 35, 5(2001), 945-980. MR.1866277 (2003a:76078)

[8] Y. Huang, Z. Shi, T. Tang and W. Xue, A multilevel successive iteration method for nonlinear elliptic problems, Math. Comp., 73, 246(2003), 525-539. MR2028418 (2004k:65206)

[9] W. Layton and W. Lenferink, Two-level Picard and modified Picard methods for the Navier-Stokes equations, Appl. Math. Comput., 69 (1995), 263-274. MR1326676 (95m:65191)

[10] W. Layton, A. Meir and P. Schmidt, A two-level discretization method for the stationary MHD equations, Electronic Transactions on Numerical Analysis, 6 (1997), 198-210. MR.1615165 (99c:76067)

[11] W. Layton and L. Tobiska, A two-level method with backtracking for the Navier-Stokes equations, SIAM J. Numer. Anal., 35, 5(1998), 2035-2054. MR.1639994 (99g:65115)

[12] M. Marion and J. Xu, Error estimates on a new nonlinear Galerkin method based on two-grid finite elements, SIAM J. Numer. Anal., 32, 4(1995), 1170-1184. MR.1342288 (96f:65136)

[13] P. Vanek, M. Brezina and R. Tezaur, Two-grid method for linear elasticity on unstructured meshes, SIAM J. Sci. Comput., 21, 3(1999), 900-923. MR.1755171(2001b:65140) 
[14] F. D. Tappert, The parabolic approximation method, In Wave Propagation and Underwater Acoustics, J. B. Keller and J. S. Papadakis (Eds.), Lecture Notes in Physics, Vol. 70, Springer-Verlag, New York, 1977. MR0475274 (57:14891)

[15] T. Utnes, Two-grid finite element formulations of the incompressible Navier-Stokes equations, Comm. Numer. Meth. Engng., 13, 8(1997), 675-684. MR.1466044(98d:76110)

[16] J. Xu and A. Zhou, Local and parallel finite element algorithms for eigenvalue problems, Acta Mathematicae Applicatea Sinica, English Series, 18, 2(2002), 185-200. MR2008551 (2004m:65184)

[17] J. Xu and A. Zhou, Local and parallel finite element algorithms based on two-grid discretizations for nonlinear problems, Adv. Comput. Math., 14, 4(2001), 293-327. MR.1865099 (2002h:65198)

[18] J. Xu and A. Zhou, Local and parallel finite element algorithms based on two-grid discretizations, Math. Comp. 69 (2000), 881-909. MR.1654026 (2000j:65102)

[19] J. Xu, Two-grid discretization techniques for linear and nonlinear PDE, SIAM J. Numer. Anal., 33, 5(1996), 1759-1777. MR1411848(97i:65169)

[20] J. Xu, A novel two-grid method for semilinear equations, SIAM J. Sci. Comput., 15, 1(1994), 231-237. MR.1257166 (94m:65178)

[21] J. Xu, A new class of iterative methods for nonselfadjoint or indefinite problems, SIAM J. Numer. Anal., 29 (1992), 303-319. MR.1154268 (92k:65063)

[22] J. Xu, Iterative methods by SPD and small subspace solvers for nonsymmetric or indefinite problems, Proceedings of the Fifth International Symposium on Domain Decomposition Methods for Partial Differential Equations, SIAM, Philadelphia, 1992. MR1189568 (93h:65080)

Institute for Computational and Applied Mathematics and Department of Mathematics, Xiangtan University, People's Republic of China

E-mail address: jjc@xtu.edu.cn

Institute for Computational and Applied Mathematics and Department of Mathematics, Xiangtan University, People's Republic of China

E-mail address: shushi@xtu.edu.cn

Institute for Computational and Applied Mathematics, Xiangtan University, PeoPle's Republic of China; and Center for Computational Mathematics and Applications, Pennsylvania State University, Pennsylvania

E-mail address: xu@math.psu.edu 